\title{
Lichens and allied fungi of the Ragusha River Protected Area (Leningrad Region, Russia)
}

\section{Ekaterina S. Kuznetsova ${ }^{1,2}$, Olga A. Kataeva ${ }^{2}$, Dmitry E. Himelbrant ${ }^{1,2}$, Jurga Motiejūnaité $\dot{e}^{3}$}

\author{
${ }^{1}$ Department of Botany, St. Petersburg State University, Universitetskaya emb. 7-9, 199034 St. Petersburg, Russia. \\ E-mails: igel_kuzn@mail.ru,d_brant@mail.ru \\ ${ }^{2}$ Laboratory of Lichenology and Bryology, Komarov Botanical Institute RAS, Professor Popov St. 2, \\ 197376 St. Petersburg, Russia. E-mail: kataevaoa@mail.ru \\ ${ }^{3}$ Laboratory of Mycology, Nature Research Centre, Institute of Botany, Žaliųjų Ežerų 49, LT-08406 Vilnius, Lithuania. \\ E-mail: jurga.motiejunaite@botanika.lt
}

\begin{abstract}
The lichen biota of the Ragusha River (protected area in Leningrad Region) is studied. In total 221 species (211 lichenized, 5 lichenicolous and 5 saprobic fungi) are listed. Lecanora perpruinosa is new to North-Western European Russia. Lathagrium fuscovirens, Pronectria erythrinella, Protoblastenia rupestris, Thelidium minutulum, T. zwackhii and Tremella hypogymniae are reported for the first time for Leningrad Region, and Ochrolechia bahusiensis for Eastern Leningrad Region. The most noteworthy part of lichen biota is the complex of 21 calcicolous lichens. Eleven of them are known in the region only from the Ragusha River valley.
\end{abstract}

Keywords: lichens, calcicolous lichens, new records, Leningrad Region, North-Western European Russia.

\section{INTRODUCTION}

The Ragusha River is the left tributary of the Volozhba River and belongs to the Syas River basin. It starts in Novgorod Region, near the village of Lushino, flows $40 \mathrm{~km}$ and joins the Volozhba River in Leningrad Region (LR). On its way, it crosses outcrops of Carboniferous limestone (Fig. 1). Here favourable conditions for karst processes have developed. In the vicinity of the village Rudnaya Gorka, the river disappears into a series of sinkholes. The length of the dry river-bed is about $2 \mathrm{~km}$, water flowing only during spring and autumn floods. Downstream the river forms a canyon with banks to $60 \mathrm{~m}$ high. Only in the last kilometer, in the Volozhba River valley, the river again changes its nature and forms numerous arms. The unique valley of the Ragusha River, between the boundary of Novgorod Region and confluence with the Volozhba River, has been protected since 1976. It occupies an area of $10.34 \mathrm{~km}^{2}$ and covers about $8 \mathrm{~km}$ of the river course (Noskov \& Botch, 1999). Vegetation consists mainly of mixed and deciduous forests, boggy in parts. Alder-elm riparian forests attract the most interest due to their rarity in Leningrad Region. Old re-vegetated clear-cut areas are common.
The lichens of the Ragusha River valley have not been explored before. The only known lichen sample (Peltigera aphthosa, now deposited in LE) from the investigated territory was collected by $\mathrm{R}$. Regel and J. Tzinserling in the surroundings of village Rudnaya Gorka in 1919 during phytosociological investigations. Our materials were collected during several field trips (since 1999 to 2007) in the Ragusha River Protected Area and its vicinities. Some parts of the collection (75

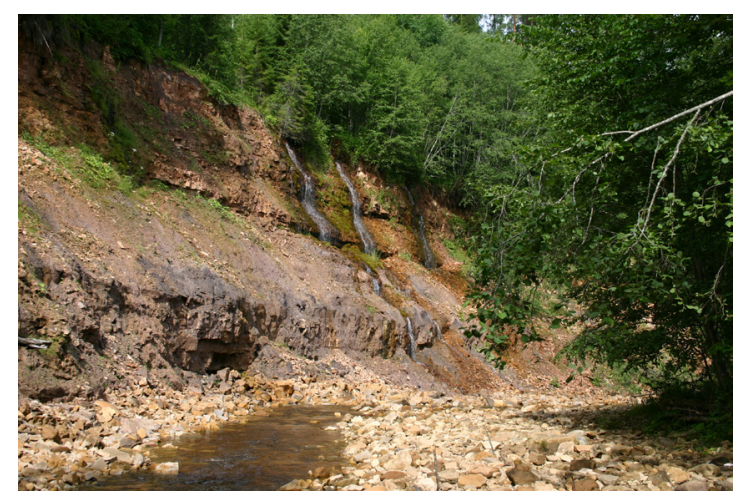

Fig. 1. Canyon of the Ragusha River (photo L. A. Basova). 
species) were identified and published previously (Kuznetsova et al., 2007, 2012; Stepanchikova et al., 2009; Pykälä et al., 2012), but mainly without indicating precise localities and substrates. This paper presents a complete annotated list of lichens and allied fungi currently known for the investigated territory.

\section{MATERIALS AND METHODS}

The materials for the present survey were collected by O. A. Kataeva on 25-26 September 1999 and by E. S. Kuznetsova on 15-22 July 2004, 16-22 July 2006 and 22-29 July 2007. Dissecting microscope, light microscope and UV light were used for the identification of lichen specimens in the laboratory. Chemical characters were studied by thin layer chromatography TLC (in solvents A, B and C) following Orange et al. (2001). Voucher specimens are deposited mainly in the herbarium of Komarov Botanical Institute RAS (LE); some specimens are stored in the herbaria of St. Petersburg State University (LECB), Botanical Museum of the University of Helsinki $(\mathrm{H})$, and Nature Research Centre, Institute of Botany in Vilnius (BILAS).

The names of the collectors or reviewers in the list of localities and species list are abbreviated as follows: DH - Dmitry E. Himelbrant, OK - Olga A. Kataeva, MK - Martin Kukwa, EK - Ekaterina S. Kuznetsova, IS - Irina S. Stepanchikova, and JP - J. Pykälä. The species included in the Red Data Book of the LR (Tzvelev, 2000) are marked with \#, those redlisted in Russian Federation (Red..., 2008) with \#\#, lichenicolous fungi with *, the saprobic fungi with + . Following abbreviations are used for substrates: Acpl - bark of Acer platanoides L., Ali - bark of Alnus incana (L.) Moench, Be - bark of Betula spp., calst - calcareous stone, dws bark of dwarf shrubs, Juc - bark of Juniperus communis L., lig - lignum, 1Ali - lignum of Alnus incana, 1Pic - lignum of Picea sp., 1Pis - lignum of Pinus sylvestris L., mos - mosses, Paa - bark of Padus avium Mill., Pic - bark of Picea sp., Pis - bark of Pinus sylvestris L., Pot - bark of Populus tremula L., Soa - bark of Sorbus aucuparia L., Ul - bark of Ulmus glabra Huds. Leningrad Region and Eastern part of Leningrad Region are abbreviated as LR and ELR, respectively.

The nomenclature of the cited taxa mainly follows Nordin et al. (2011). The species in the list are accompanied by locality information, substrates, herbaria and references. Most species were identified by the authors; otherwise special notes are made.

\section{List of localities}

Protected area: $1-59^{\circ} 15^{\prime} 18^{\prime \prime} \mathrm{N}, 33^{\circ} 56^{\prime} 49^{\prime \prime} \mathrm{E}$, aspen forest with young spruces, EK, 17.07.2006; $2-59^{\circ} 15^{\prime} 26^{\prime \prime} \mathrm{N}, 33^{\circ} 56^{\prime} 30^{\prime \prime} \mathrm{E}$, mixed forest, EK, 17.07.2006; $3-59^{\circ} 16^{\prime} \mathrm{N}, 33^{\circ} 55^{\prime} \mathrm{E}$, elm forest in the canyon, OK, 25.09.1999, EK, 24.06.2007; $4-59^{\circ} 16^{\prime} 06^{\prime \prime} \mathrm{N}, 33^{\circ} 55^{\prime} 47^{\prime \prime} \mathrm{E}$, riverside spruce forest with young birches, grey alders and rowans, EK, 16.07.2006; $5.1-59^{\circ} 16^{\prime} 13^{\prime \prime} \mathrm{N}$, $33^{\circ} 56^{\prime} 12$ "E, young spruce forest with aspens, EK, 16.07.2006; 5.2 - same place, road in the old clear-cut area, EK, 16.07.2006; 6-59¹6’15”N, $33^{\circ} 56^{\prime} 45^{\prime} \mathrm{E}$, re-vegetated clear-cut areas, EK, 18.07.2006; 7 - 59 $9^{\circ} 16^{\prime} 16^{\prime \prime} \mathrm{N}, 33^{\circ} 55^{\prime} 59^{\prime \prime} \mathrm{E}$, riverside young spruce forest with aspens, rowans and very old birches, EK, 18.07.2006; 8 $59^{\circ} 16^{\prime} 20^{\prime \prime} \mathrm{N}, 33^{\circ} 55^{\prime} 57^{\prime \prime} \mathrm{E}$, riverside young spruce forest with young grey alders, EK, 16.07.2006; 9 - 59 $16^{\prime} 21^{\prime \prime} \mathrm{N}, 33^{\circ} 55^{\prime} 42^{\prime} \mathrm{E}$, young deciduous forest, EK, 17.07.2004; $10-59^{\circ} 16^{\prime} 45^{\prime \prime} \mathrm{N}$, $33^{\circ} 55^{\prime} 53^{\prime \prime} \mathrm{E}$, river bank and elm forest on the river valley slope, EK, 19.07.2006; $11-59^{\circ} 16^{\prime} 49^{\prime \prime} \mathrm{N}$, $33^{\circ} 55^{\prime} 38^{\prime \prime} \mathrm{E}$, elm forest in the canyon, EK, 19.07.2004; 12 - 59 $16^{\prime} 50 ” \mathrm{~N}, 33^{\circ} 55^{\prime} 38^{\prime \prime} \mathrm{E}$, elm forest in the canyon, EK, 25.06.2007; $13.1-59^{\circ} 17^{\prime} 03^{\prime \prime} \mathrm{N}, 33^{\circ} 55^{\prime} 13^{\prime \prime} \mathrm{E}$, elm forest with grey alder in the canyon, OK, 25.09.1999, EK, 19.07.2004, 06.2007; 13.2 - same place, steep slope of the canyon, OK, 25.09.1999, EK, 19.07.2004; 13.3 - same place, young spruce forest, OK, 25.09.1999; 14 - 59¹6'59”N, $33^{\circ} 55^{\prime} 27^{\prime \prime} \mathrm{E}$, elm forest in the canyon, EK, 19.07.2006; 15 - 59 $17^{\prime} 32^{\prime \prime N}, 33^{\circ} 54^{\prime} 15^{\prime} \mathrm{E}$, old clear-cut area with spruces, aspens and rowans, EK, 19.07.2006; 16 - 59 18 '05”N, 3355'37”E, riverside deciduous forest, EK, 20.07.2004; $17.1-59^{\circ} 16^{\prime} \mathrm{N}, 33^{\circ} 55^{\prime} \mathrm{E}$, mixed forest in the canyon, OK, 25.09.1999; 17.2 - same place, young elm forest in the canyon, EK, 06.2007; 18 $-59^{\circ} 16^{\prime} 16^{\prime \prime} \mathrm{N}, 33^{\circ} 55^{\prime} 48^{\prime \prime} \mathrm{E}$, deciduous forest, EK, 18.07.2006; $19-59^{\circ} 16^{\prime} 22^{\prime \prime} \mathrm{N}, 33^{\circ} 55^{\prime} 59^{\prime \prime} \mathrm{E}$, riverside pine forest with birches, EK, 17.07.2004; $20-59^{\circ} 16^{\prime} 26^{\prime \prime} \mathrm{N}, 33^{\circ} 56^{\prime} 25^{\prime \prime} \mathrm{E}$, bog with aspens, spruces and birches, OK, 25.09.1999; 21 $59^{\circ} 16^{\prime} 34^{\prime \prime} \mathrm{N}, 33^{\circ} 55^{\prime} 53^{\prime \prime} \mathrm{E}$, deciduous forest in the canyon, EK, 19.07.2006; $22-59^{\circ} 16^{\prime} 34^{\prime \prime} \mathrm{N}$, $33^{\circ} 56^{\prime} 32$ "E, mixed forest, EK, 20.07.2004, 
$06.2007 ; 23$ - 59 $16^{\prime} 36^{\prime \prime} \mathrm{N}, 33^{\circ} 56^{\prime} 2^{\prime \prime} \mathrm{E}$, pine forest with spruces, OK, 26.09.1999; 24 $59^{\circ} 16^{\prime} 37^{\prime \prime N} 33^{\circ} 55^{\prime} 44^{\prime \prime} \mathrm{E}$, birch forest with young spruces and willows, OK, 25.09.1999; $25-59^{\circ} 16^{\prime} 37^{\prime \prime} \mathrm{N} 33^{\circ} 56^{\prime} 50^{\prime \prime} \mathrm{E}$, pine forest, OK,

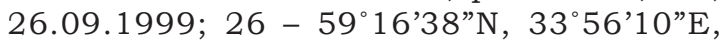
pine forest, OK, 26.09.1999; $27-59^{\circ} 16^{\prime} 38^{\prime \prime} \mathrm{N}$, $33^{\circ} 56^{\prime} 80$ ”'E, swamp pine forest, OK, 26.09.1999; $28-59^{\circ} 16^{\prime} 40^{\prime \prime} \mathrm{N}, 33^{\circ} 56^{\prime} 12^{\prime \prime} \mathrm{E}$, pine forest, OK,

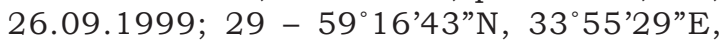
birch forest with aspens and young spruces, OK, 25.09.1999; 30 - 59 $16^{\circ} 46^{\prime \prime N} 33^{\circ} 55^{\prime} 23^{\prime \prime} \mathrm{E}$, aspen forest with spruces and a few birches,

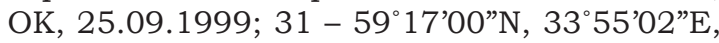
roadside aspens, EK, 20.07.2004. Vicinities of protected area: $32-59^{\circ} 15^{\prime} 45^{\prime \prime} \mathrm{N}, 33^{\circ} 56^{\prime} 42^{\prime \prime} \mathrm{E}$, aspen forest with spruces, EK, 18.07.2006; 33 - 59 $16^{\circ} 02^{\prime \prime} \mathrm{N}, 33^{\circ} 56^{\prime} 52^{\prime \prime} \mathrm{E}$, swamp pine forest, EK, 18.07.2006; $34-59^{\circ} 16^{\prime} 03^{\prime \prime} \mathrm{N}, 33^{\circ} 57^{\prime} 51^{\prime \prime} \mathrm{E}$, fringe of mixed forest, EK, 24.06.2007; 35 - 59 $16^{\prime} 21^{\prime \prime} \mathrm{N}, 33^{\circ} 56^{\prime} 35^{\prime \prime} \mathrm{E}$, mixed forest, EK, 17-18.07.2004; $36-59^{\circ} 16^{\prime} 26^{\prime \prime} \mathrm{N}, 33^{\circ} 57^{\prime} 12^{\prime \prime} \mathrm{E}$, spruce forest, OK, 26.09.1999; $37-59^{\circ} 16^{\prime} 29^{\prime \prime} \mathrm{N}$, $33^{\circ} 57$ '20" $\mathrm{E}$, spruce forest, OK, 26.09.1999; $38-59^{\circ} 16^{\prime} 32^{\prime \prime} \mathrm{N}, 33^{\circ} 56^{\prime} 37^{\prime \prime}$, spruce forest, OK, 26.09.1999; $39-59^{\circ} 16^{\prime} 39-41^{\prime \prime} \mathrm{N}, 33^{\circ} 57^{\prime} 19-20^{\prime \prime} \mathrm{E}$, vicinity of the village, OK, 26.09.1999; 40 $59^{\circ} 16^{\prime} 41^{\prime \prime} \mathrm{N}, 33^{\circ} 57^{\prime} 19^{\prime \prime} \mathrm{E}$, quarry in the vicinity of the village, OK, 26.09.1999.

\section{List of species}

ACROCORDIA CAVATA (Ach.) R. C. Harris - 3: old Ul (Stepanchikova et al., 2009).

\#Alectoria sarmentosa (Ach.) Ach. subsp. SARMEnTOSA - 22, 33: Pis (Kuznetsova et al., 2007).

AlyXoria VARIA (Pers.) Ertz \& Tehler - 3, 4: old Ul, Pot.

ANISOMERIDIUM POLYPORI (Ellis \& Everh.) M. E. Barr - 12: mossy bark of Ul.

Arthonia apatetica (A. Massal.) Th. Fr. - 20: Pot. ARTHONia DidYMa Körb. - 4: Pot.

Arthonia helvola (Nyl.) Nyl. - 17.1: lig (rotten stump).

Arthonia RAdiata (Pers.) Ach. - 13.1, 14, 16, 31: Ali, Acpl.

ARthonia RUANA A. Massal. - 13.1, 16: Ali, Ul. Athallia PYRacea (Ach.) Arup, Frödén \& Søchting - 9, 15: Pot, lig (Kuznetsova et al., 2007).

Bacidia arceutina (Ach.) Arnold - 2, 32: Pot.

Bacidia bagliettoana (A. Massal. \& De Not.) Jatta - 5.1, 9: Pot.
Bacidia CiRcumspecta (Nyl. ex Vain.) Malme - 4: Pot.

BaCidia IgNiaRi (Nyl.) Oxner - 20: Pot.

Bacidia Rubella (Hoffm.) A. Massal. - 3: old U1 (Kuznetsova et al., 2007).

BACIDIA Subincompta (Nyl.) Arnold - 2, 13.1, 16: Ali, Pot, Ul.

BAEOMYCES CARNEUS Flörke - 5.2: soil on roadside. Biatora Albohyalina (Nyl.) Bagl. \& Carestia - 20: Pot.

BiATORA EFFLORESCENS (Hedl.) Räsänen - 16, 17.1: bark of deciduous tree, Pic. Specimen from loc. 16 contains argopsin, norargopsin and 3 unidentified yellow pigments, det. MK \& IS, 10.2011.

Biatora Globulosa (Flörke) Fr. - 20: Pot (Kuznetsova et al., 2007).

BiatORA HElvola Körb. ex Hellb. - 9, 10, 19, 22, 32, 35: Ali, Pic, Pis, Pot (Kuznetsova et al., 2007).

Biatora OCElliformis (Nyl.) Arnold - 10: Ali, Paa. Bilimbia microcarpa (Th. Fr.) Th. Fr. - 5.1: Pot.

Bilimbia SABuletorum (Schreb.) Arnold - 13.1, 14, 39: lig, mos, calcareus soil.

BRYORIA CAPILLARIS (Ach.) Brodo \& D. Hawksw. 5.1, 8, 20, 22, 24, 33, 37, 38: Be, Pic, Pis, Pot. Some specimens were identified by I. Brodo (Kuznetsova et al., 2007).

Bryoria furcellata (Fr.) Brodo \& D. Hawksw. - 33: Pis.

BRYORIA FUSCESCENS (Gyeln.) Brodo \& D. Hawksw. - 8, 5.1, 9, 19, 20, 24, 25, 28, 32, 35,: Be, Pic, Pis, Pot. Some specimens were identified by I. Brodo (Kuznetsova et al., 2007).

Bryoria IMPLEXA (Hoffm.) Brodo \& D. Hawksw. - 40: Pis.

\#BRyoria NADVORniKiana (Gyeln.) Brodo \& D. Hawksw. - 20, 24, 37: Ali, Be, Pic. Some specimens were identified by I. Brodo (Kuznetsova et al., 2007).

Bryoria Simplicior (Vain.) Brodo \& D. Hawksw. - 22: Pic.

Buellia Disciformis (Fr.) Mudd - 3, 10, 13.1, 14, 20: Ali, Paa, Pot, U1.

Buellia erubescens Arnold - 9, 13.1, 14, 16, 20, 22, 35: bark of deciduous trees.

BuElLIA GRISEOVIRENS (Turner \& Borrer ex Sm.) Almb. - 14, 16: Ali, Ul. Specimen from loc. 16 contains atranorin and norstictic acid, det. MK \& IS, 10.2011.

Calicium glaucellum Ach. - 33: 1Pis.

Calicium trabinellum (Ach.) Ach. - 9, 20, 33, 35: Ali, Pis, lig (Kuznetsova et al., 2007). 
CAlicium Viride Pers. - 35: dead Pis.

Caloplaca Cerina (Hedw.) Th. Fr. - 1, 9, 15, 20, 31: Ali, lig, Pot (Kuznetsova et al., 2007).

CANDElariella lutella (Vain.) Räsänen - 5.1, 9, 20: Pot. Specimen from loc. 5.1 det. by L. S. Yakovchenko \& M. Westberg, 2012.

Catillaria nigroclavata (Nyl.) Schuler $-5.1,9$, 15, 20: lig, Pot.

Catinaria atropurpurea (Schaer.) Vězda \& Poelt - 20: Pot.

CAtinaRia NeUschildi (Körb.) P. James - 20: dead Pot.

Cetraria islandica (L.) Ach. subsp. ISLAndica - 6, 33: soil (Kuznetsova et al., 2007).

Cetraria sepincola (Ehrh.) Ach. - 9, 33: Be, Pis, Pot (Kuznetsova et al., 2007).

CHAENOTHECA BRACHYPODA (Ach.) Tibell - 3: old Ul.

ChAENothecA CHRYsocephala (Turner ex Ach.) Th. Fr. - 5.1, 19: Pic.

Chaenotheca ferruginea (Turner ex Sm.) Mig. 4, 5.1, 19, 22, 32, 33, 35: Pic, rarely Pis and 1Pis (Kuznetsova et al., 2007).

Chaenotheca FuRfuracea (L.) Tibell $-7,12$ : soil, lig (upturned roots).

Chaenotheca trichialis (Ach.) Th. Fr. -7 , 9: Be, 1 Pic.

Cladonia arbuscula subsp. Beringiana Ahti 3, 9: lig, soil.

Cladonia ARBuscula subsp. mitis (Sandst.) Ruoss - 6, 33: soil, Pis (Kuznetsova et al., 2007).

Cladonia botrytes (K. G. Hagen) Willd. - 3, 6, 9, 33: lig, soil, Pis (Kuznetsova et al., 2007).

Cladonia Cariosa (Ach.) Spreng. - 39: soil.

Cladonia Carneola (Fr.) Fr. - 6, 11, 33: lig, mossy soil, Pis.

Cladonia cenotea (Ach.) Schaer. - 2, 3, 6, 15, 23, 33, 35: lig, soil, bark of trees (Kuznetsova et al., 2007).

Cladonia chlorophaea (Flörke ex Sommerf.) Spreng. s. 1. - 3, 5.1, 6, 7, 13.1, 14, 15, 17.1, 19, 23, 32, 33, 35, 40: lig, soil, bark of various trees (Kuznetsova et al., 2007).

Cladonia Chlorophaea (Flörke ex Sommerf.) Spreng. s. str. - 19: Pic.

Cladonia coniocraea (Flörke) Spreng. - 2-4, $5.1,6,7,9,10,12,13.1,15,16,22,23$, 33, 35: lig, soil, bark of trees (Kuznetsova et al., 2007).

Cladonia cornuta (L.) Hoffm. subsp. cornuta 2, 6, 33: soil, Be (Kuznetsova et al., 2007).

Cladonia CRISPATA (Ach.) Flot. var. CRISPATA - 3: lig. Cladonia digitata (L.) Hoffm. - 2, 9: Be, lig.
Cladonia fimbriata (L.) Fr. - 16, 33, 39, 40: lig, soil, Ali, Pis.

Cladonia Furcata (Huds.) Schrad. - 3: lig.

Cladonia gracilis (L.) Willd. - 3, 6, 9: lig, soil.

Cladonia macilenta Hoffm. - 9: soil.

Cladonia norvegica Tønsberg \& Holien - 9: lig.

Cladonia Pleurota (Flörke) Schaer. -5.2 , 6: soil.

Cladonia Pyxidata (L.) Hoffm. - 13.1, 13.2: sandy soil, plant debris.

Cladonia Rangiferina (L.) F. H. Wigg. - 3, 6, 33: lig, soil (Kuznetsova et al., 2007).

Cladonia Rei Schaer. - 3, 17.1, 33, 39: Be, lig, soil.

Cladonia stygia (Fr.) Ruoss - 33: soil (Kuznetsova et al., 2007).

Cladonia SUlPhURINa (Michx.) Fr. - 2, 33: soil, Be, Pis (Kuznetsova et al., 2007).

Cladonia uncialis (L.) Weber ex F. H. Wigg. subsp. UNCIALIS - 33: soil (Kuznetsova et al., 2007).

Cliostomum leprosum (Räsänen) Holien \& Tønsberg - 2, 33: Pic, 1Pis.

COENOGONIUM PINETI (Ach.) Lücking \& Lumbsch - 17.1: lig, U1.

Collema Flaccidum (Ach.) Ach. - 13.2: mossy soil.

\#COllema fURfuraceum (Arnold) Du Rietz - 1, 13.1: Pot.

\#Collema subnigrescens Degel. - 1, 4, 15, 32, 34: Pot.

*DActylospora Deminuta (Th. Fr.) Triebel - 5.1: thallus of Mycobilimbia sp.

Enchylium tenaX (Sw.) Gray - 13.2: mossy calcareus soil.

EVERNIA MESOMORPHA Nyl. - 5.1, 13.1, 13.3, 20, 22, 28, 33, 35-37: bark of trees (Kuznetsova et al., 2007).

EVERNIA PRUNASTRI (L.) Ach. - 2, 4, 9, 10, 14, 16, 19, 20, 35: bark of deciduous trees, Pic (Kuznetsova et al., 2007).

Fuscidea ARBORICOla Coppins \& Tønsberg - 4, 22, 32: 1Pic (twigs). All specimens contain fumarprotocetraric acid, det. MK \& IS, 10.2011.

Fuscidea Pusilla Tønsberg - 5.1, 9, 16, 32: Ali, Pic, 1Pic.

GraPHIS SCRIPTA (L.) Ach. s. 1. - 3, 10, 13.1, $14,16,17.1,22$ : bark of deciduous trees (Kuznetsova et al., 2007).

Gyalecta fagicola (Arnold) Kremp.- 20: Pot.

Gyalecta truncigena (Ach.) Hepp - 32: Pot.

HyPOGYMnia PHYSODES (L.) Nyl. - 1-4, 5.1, 8, 9, $13.1,13.3,14,16,19-22,24,25,28,32$, 33, 35-37: bark of trees, lig (Kuznetsova et al., 2007). 
Hypogymnia tubulosa (Schaer.) Hav. - 4, 5.1, 8-10, $13.319,20,22,25,28,32,33,35-37$ : bark of trees (Kuznetsova et al., 2007).

Hypogymnia vitTata (Ach.) Parrique - 2, 30: Be.

IMSHAUGia ALEURITES (Ach.) S. L. F. Meyer - 33, 35: Pis, 1Pis, Soa (Kuznetsova et al., 2007). JAPEWIA SUBAURIFERA Muhr \& Tønsberg - 2: Pic. JAPEWIA TORnOEnsis (Nyl.) Tønsberg - 24, 38: Pic (twigs) (Kuznetsova et al., 2007).

LATHAGRIUM FUSCOVIRENS (With.) Otálora et al. - 13.2: calst. New to LR. Distribution in North-Western European Russia outside of LR: Republic of Karelia (Fadeeva et al., 2007). Distribution in Fennoscandia and Baltic countries: Norway, Sweden, Finland (Nordin et al., 2011), Estonia (Randlane et al., 2015), Latvia (Ābolinga et al., 2015), Lithuania (Motiejūnaitè, 1999).

LeCANia CYRTElla (Ach.) Th. Fr. - 9, 13.1, 20, 31: Ali, Pot, U1.

LeCANia CYRTELlina (Nyl.) Sandst. - 13.1, 20: Pot, U1.

LECANIA NAEGELII (Hepp) Diederich \& van den Boom - 5.1, 9, 14-16, 20, 31: Ali, Pot, Soa, lig.

LECANIA SYLVESTRIS (Arnold) Arnold - 13.1: calst.

LeCANORA Albellula (Nyl.) Th. Fr. - 31: Ali.

LECANORA ALlOPHANA Nyl. - 1, 2, 20, 31, 32: Ali, Pot.

Lecanora argentata (Ach.) Malme - 20: Pot.

LECANORA CARPINEA (L.) Vain. - 9, 14, 16: Acpl, Ali, Pot, Soa.

Lecanora cateilea (Ach.) A. Massal. - 20: Pot.

LECANORA CHLAROTERA Nyl. - 7-9, 10, 13.1, 14, 20-22, 39: bark of deciduous trees, Pic (Kuznetsova et al., 2007).

LECANORA cf. COMPAlLENS Herk \& Aptroot - 20: dead Pot. Specimen contains usnic acid and zeorin, det. IS, 29.02.2016 (LE L-13132).

LECANORA CRENULATA Hook. - 13.2: calst (Kuznetsova et al., 2007).

LECANORA DISPERSA (Pers.) Sommerf. - 13.2: calst on the river banks or submerged in water.

LECANORA HAGENII (Ach.) Ach. - 9: Pot.

LECANORA HYPOPTA (Ach.) Vain. - 13.1, 33, 35: Pic, 1Pic, 1Pis, plant debris.

LECANora norvegica Tønsberg - 5.1, 33: Pic, Pis. Both specimens contain protocetraric acid, in specimens from loc. 5.1 atranorin and fatty acid (trace) were also detected, det. MK \& IS, 10.2011.

LECANORA PERPRUINOSA Fröberg - 13.2: calst. New to North-Western Europan Russia. Distribution in European Russia outside of LR:
Republic of Komi (Hermansson et al., 2006), Tver' Region (Notov, 2011), Ryazan' Region (Muchnink \& Śliwa, 2011). Distribution in Fennoscandia and Baltic countries: Norway, Sweden, Finland (Nordin et al., 2011), Estonia (Randlane et al., 2015).

LECANORA PULICARIS (Pers.) Ach. - 9, 13.1, 14, 16, 20, 35: Ali, Pot, Soa.

Lecanora symmicta (Ach.) Ach. - 5.1, 8, 9, 13.1, 14, 16, 20, 22, 32: bark of trees, Pic (Kuznetsova et al., 2007).

LeCANORA UMBrina - 9: Pot (Kuznetsova et al., 2007).

LECIDEA ERYTHROPHAEA Flörke ex Sommerf. - 1, 2, 20: Pot (Kuznetsova et al., 2007).

LECIDEA LEPRARIOIDES Tønsberg - 4: Pic (twigs). Specimen contains pseudoplacodiolic acid, det. MK \& IS, 10.2011 .

LECIDEA NYLANDERI (Anzi) Th. Fr. - 2, 5.1, 7, 10, 15, 19, 20, 22, 24, 25, 32, 33, 35, 37: Ali, $\mathrm{Be}$, Pot, bark of coniferous trees (Kuznetsova et al., 2007).

LECIDEA TURGIDULA Fr. - 5.1, 9, 19, 22, 32, 33, 35: Pic, 1Pic, Pis, 1Pis.

Lecidella elaeochroma (Ach.) M. Choisy - 1, 5.1, 7, 9, 16, 31, 32: Ali, Pot.

LECIDELLA EUPHOREA (Flörke) Hertel - 31: Ali.

LEPRARIA EBURNEA J. R. Laundon - 14: mos. Specimen contains alectorialic acid, det. MK \& IS, 10.2011.

LePRARIA InCANA (L.) Ach. - 2, 15, 17.1: Pic, Ul (stump and roots).

LEPRARIA JACKII Tønsberg - 4, 5.1, 9, 32: Pic, 1Pic, Pis. All specimens contain atranorin, jackinic/rangiformic and roccellic acids, in specimens from loc. 9 and 32 norjackinic/ norrangiformic acids were identified, det. MK \& IS, 10.2011.

LEPRARIA LOBIFICANS Nyl. $-7,13.1$ : soil, lig, plant debris.

LEPTOGIUM SATURNinum (Dicks.) Nyl. - 2, 4, 5.1, 32: Pot.

LEPTORHAPHIS ATOMARIA (Ach.) Szatala - 9: Pot.

+LePtorhaphis EPIDERMidis (Ach.) Th. Fr. - 9: Be (twigs).

* Lichenostigma maureri Hafellner - 8: thallus of Bryoria capillaris on twigs of Pic, det. MK, 10.2011 (Kuznetsova et al., 2012).

\#\#Lobaria pulmonaria (L.) Hoffm. - 4, 30: Pot (Kuznetsova et al., 2007).

Loxospora elatina (Ach.) A. Massal. - 5.1: Pis. Specimen contains thamnolic and elatinic acids, det. MK \& IS, 10.2011. 
Melanelixia Glabratula (Lamy) Sandler \& Arup - 13.1: Ali.

Melanelixia subaurifera (Nyl.) O. Blanco et al. 4, 10, 12, 13.1, 14, 16, 20: Acpl, Ali, Paa, Pot, Pic.

Melanohalea EXasperata (De Not.) O. Blanco et al. - 5.1: Pot.

Melanohalea exasperatula (Nyl.) O. Blanco et al. - 13.1, 20, 21, 32: Juc, Paa, Pot, Pic.

Melanohalea olivacea (L.) O. Blanco et al. - 4, 8, 9, 20: Be, Pic, Pot, Soa (Kuznetsova et al., 2007).

Melanohalea SePtentrionalis (Lynge) O. Blanco et al. - 13.3: Juc.

Micarea melaena (Nyl.) Hedl. - 5.1: Pis.

MicAREA NiTSCHKEANA (J. Lahm ex Rabenh.) Harm. - 22: Pis (Kuznetsova et al., 2007).

MicAREA PRASINA Fr. s.1. - 5.1, 19, 22, 24, 36: Pic, Pis (Kuznetsova et al., 2007).

Multiclavula CoRYnoides (Peck) R. H. Petersen vicinity of Rudnaya Gorka, pine forest with spruces and birches, soil, 07.2001, leg. \& det. V. M. Kotkova (LE 212137) (Kotkova, 2003).

Mycobilimbia carneoalbida (Müll. Arg.) Printzen $-2,4,5.1,15,32$ : Pot (mossy bark).

Mycobilimbia EPIXANTHOIDES (Nyl.) Vitik. et al. 15: Pot.

Mycobilimbia tetramera (De Not.) Vitik. et al. ex Hafellner \& Türk - 1: Pot.

Mycoblastus alpinus (Fr.) Th. Fr. ex Hellb. - 2: Be. Mycoblastus SANGUinarius (L.) Norman - 33: Pis.

+Mycocalicium subtile (Pers.) Szatala - 9, 13.1, 14, 16, 22, 33, 35: 1Ali, Pic, Pis.

Naetrocymbe punctiformis (Pers.) R. C. Harris - 9: Be.

Nephroma Parile (Ach.) Ach. - 4: Pot.

OCHROLECHIA ARBOREA (Kreyer) Almb. - 20: dead Pot. Specimen contains gyrophoric and lecanoric acids and lichexanthone, det. IS, 29.02.2016 (LE L-13128).

OChrolechia BahUsiensis H. Magn. - 10: Paa, Ul. Specimen contains gyrophoric, lecanoric acids and murolic acid complex, det. MK \& IS, 10.2011. New to ELR.

OCHROLECHIA MICROSTICTOIDES Räsänen - 2, 35: $\mathrm{Be}$, Pis.

Ochrolechia Pallescens (L.) A. Massal. - 20: dead Pot. Specimens contain variolaric acid and murolic acid complex in thalli, gyrophoric and lecanoric acids in epihymenium, det. IS, 29.02.2016 (LE L-13130, L-13131).
Palicella filamentosa (Stirt.) Rodr. Flakus \& Printzen - 33: 1Pis.

PARMelia sulcata Taylor $-1,4,8-10,13.1,14$, 16, 19, 21, 35: bark of deciduous trees, Pic (Kuznetsova et al., 2007).

Parmeliopsis Ambigua (Wulfen) Nyl. - 5.1, 7, 8, $10,13.3,20,22,24,25,28,32,33,35-37$ : bark of deciduous and coniferous trees, dws (Ledum palustre, Vaccinium uliginosum) (Kuznetsova et al., 2007).

PARMELIOPSIS HYPEROPTA (Ach.) Arnold - 25, 28, 33, 35, 37: Pic, Pis, dws (Ledum palustre, Vaccinium uliginosum) (Kuznetsova et al., 2007).

Peltigera aphthosa (L.) Willd. - 6, 22, 23: soil, Juc (mossy bark) (Kuznetsova et al., 2007).

Peltigera DidACTYla (With.) J. R. Laundon - 19: soil.

Peltigera extenuata (Nyl. ex Vain.) Lojka - 7, 39: soil.

Peltigera NECKeri Hepp ex Müll. Arg. - 12: mossy bark, det. O. Vitikainen, 2008.

Peltigera neOPOLYDACTYla (Gyeln.) Gyeln. - 3: Ali (fallen tree).

Peltigera polydactylon (Neck.) Hoffm. - 3, 14, 19, 24: Ali (fallen tree), Pic, soil.

Peltigera PRAetextata (Flörke ex Sommerf.) Zopf $-1-4,5.1,7,9,13.1,14,15,17.1,19,29$, 32: mossy base of deciduous trees, lig, sandy soil (Kuznetsova et al., 2007).

Peltigera Rufescens (Weiss) Humb. - 13.1, 39 : Ali (mossy bark), soil.

Pertusaria albescens (Huds.) M. Choisy \& Werner - 10, 15, 16: Ali, Pot (Kuznetsova et al., 2007).

Pertusaria alpina Hepp ex Ahles - 13.1: Ali. Pertusaria amara (Ach.) Nyl. - 2, 32: Be, Pot.

PhaEophyscia Endophoenicea (Harm.) Moberg 13.1, 17.2: U1 (Stepanchikova et al., 2009).

Phlyctis ARgena (Spreng.) Flot. - 1, 2-4, 5.1, 10, 13.1, 15, 32: Ali, Pot, U1, Juc.

Physcia adscendens H. Olivier - 1, 9, 14, 16, 31 : Acpl, Ali, Pot (Kuznetsova et al., 2007).

Physcia Aipolia (Ehrh. ex Humb.) Fürnr. - 5.1, 9, 15, 20, 22, 31, 39: Ali, Pot, Soa, lig (Kuznetsova et al., 2007).

Physcia AlNophila (Vain.) Lohtander et al. - 14, 20: Ali, Pot (Kuznetsova et al., 2007).

PhYSCONIA DISTORTA (With.) J. R. Laundon - 1, 9, 14, 20, 31, 32, 39: Ali, Pot (Kuznetsova et al., 2007).

Placynthiella icmalea (Ach.) Coppins \& P. James $-3,12,17.1,23$ : lig, mos, Juc. 
Placynthiella oligotropha (J. R. Laundon) Coppins \& P. James - 6: soil.

Placynthiella uliginosa (Schrad.) Coppins \& P. James - 13.1: lig.

Platismatia glauca (L.) W. L. Culb. \& C. F. Culb. $-2,4,5.1,7-9,17.1,19,20,22,25,28,32$, 33, 35-37: Be, Pic, Pis, Pot, Pic (Kuznetsova et al., 2007).

*Pronectria erythrinella (Nyl.) Lowen - 39, thallus of Peltigera extenuata (BILAS). New to LR. Distribution in North-Western European Russia outside of LR: Republic of Karelia (Fadeeva et al., 2007). Distribution in Fennoscandia and Baltic countries: Norway, Sweden, Finland (Nordin et al., 2011), Estonia (Randlane et al., 2015), Lithuania (Motiejūnaitè, 1999).

Protoblastenia RUPESTRIS (Scop.) J. Steiner 13.2, calst. New to LR. Reported from LR erroneously by A. Zavarzin with colleagues (Zavarzin et al., 1999: 249) on the basis of incorrect citation of synonym (Wainio, 1878: 54). Known from St. Petersburg (Stepanchikova et al., 2016). Distribution in North-Western European Russia outside of LR: Republic of Karelia (Fadeeva et al., 2007). Distribution in Fennoscandia and Baltic countries: Norway, Sweden, Finland (Nordin et al., 2011), Estonia (Randlane et al., 2015), Latvia (Ābolina et al., 2015).

PseudeVernia Furfuracea (L.) Zopf - 5.1, 19, 22, 25, 33, 35, 37: Pic, Pis (Kuznetsova et al., 2007).

Pseudoschismatomma Rufescens (Pers.) Ertz \& Tehler - 4: Pot.

Pycnora leucococca (R. Sant.) R. Sant. - 9, 22, 37: Pic, Pis (Kuznetsova et al., 2007).

PYCNORA SOROPHORA (Vain.) Hafellner - 33: Pis, 1Pis.

\#Ramalina dilacerata (Hoffm.) Hoffm. - 20, 21 : Ali, Paa, Pot (Kuznetsova et al., 2007).

Ramalina farinacea (L.) Ach. - 1, 20: Pot, (LE L-13129).

Ramalina sinensis Jatta - 2, 9, 18, 20, 22: Pot.

RAMBOLDIA CINNABARINA (Sommerf.) Kalb, Lumbsch \& Elix - 10: Paa.

Rinodina laevigata (Ach.) Malme - 20: Pot.

Rinodina sophodes (Ach.) A. Massal. - 20: Pot.

ROPALOSPORA VIRIDIS (Tønsberg) Tønsberg - 5.1, 10, 13.1, 16: Ali, Paa, Pic, Ul. Specimens contain perlatolic acid, det. MK \& IS, 10.2011 .

SARCOGYNe REGULARIS Körb. - 13.2: calst.
+SAREa difformis (Fr.) Fr. - 35: resin of Pis.

+SAREa Resinae (Fr.: Fr.) Kuntze - 19: resin of Pic (Kuznetsova et al., 2007).

Sclerophora Pallida (Pers.) Y. J. Yao \& Spooner - 3: U1 (Stepanchikova et al., 2009).

SCOLICIOSPORUM SAROTHAMnI (Vain.) Vězda - 9: Soa. \#SCytinium lichenoides (L.) Otálora et al. - 13.1: mos, soil (Kuznetsova et al., 2007).

SCytinium teretiusculum (Wallr.) Otálora et al. - 7: Pot.

+Stenocybe pullatula (Ach.) Stein - 13.1, 14, 16, 31: Ali.

THELIDIUM APHANES J. Lahm - 14: calst, det. JP, 2011 (H; Pykälä et al., 2012).

Thelidium Fontigenum A. Massal. - 13.2, 14: calst, det. JP, 2011 (H; Pykälä et al., 2012).

Thelidium cf. FUlloËnse Servit - 14: calst, det. JP, 2011 (H, sub T. aphanes).

Thelidium minimum (A. Massal. ex Körb.) Arnold - 13.2: calst, det. JP, 2011 (H; Pykälä et al., 2012).

Thelidium Minutulum Körb. - 10: submerged calst. New to LR, known from St. Petersburg (Pykälä et al., 2012). Distribution in NorthWestern European Russia outside of LR: Novgorod Region (Kataeva, 2009). Distribution in Fennoscandia and Baltic countries: Finland, Norway, Sweden (Nordin et al., 2011), Estonia (Randlane et al., 2011), and Lithuania (Motiejūnaite, 2002).

THELIDIUM ZWACKHII (Hepp) A. Massal. - 13.2: calst. New to LR. Distribution in NorthWestern European Russia outside of LR: Novgorod Region (Kataeva, 2009). Distribution in Fennoscandia and Baltic countries: Norway, Sweden, Finland (Nordin et al., 2011), Estonia (Randlane et al., 2015), Latvia (Āboliņa et al., 2015), and Lithuania (Motiejūnaitè, 1999).

TRAPELIOPSIS GRANUlOSA (Hoffm.) Lumbsch - 6, 23: soil, Juc.

*Tremella hypogymniae Diederich \& M. S. Christ. - loc. 39: thallus of Hypogymnia physodes (BILAS). New to LR. Distribution in NorthWestern European Russia outside of LR: Republic of Karelia (Fadeeva et al., 2007). Distribution in Fennoscandia and Baltic countries: Norway, Sweden, Finland (Nordin et al., 2011), Estonia (Randlane et al., 2015), Latvia (Motiejūnaitė \& Grochowski, 2014), and Lithuania (Motiejūnaite \& Andersson, 2003). 
TuCKERMANNOPSIS CHLOROPHYLLA (Willd.) Hale - 5.1, 8, 9, 13.3, 19, 20, 22, 25, 28, 32, 35, 36, 37: Juc, Pic, 1Pic, Pot, Pis, 1Pis (Kuznetsova et al., 2007).

UsNeA DASOPOGA (Ach.) Nyl. - 22, 25, 35-37: Be, Pic.

UsNeA GLABRescens (Nyl. ex Vain.) Vain. ex Räsänen - 37: Pic.

UsNeA HIRTA (L.) Weber ex F. H. Wigg. - 22, 33, 35: Pic, Pis (Kuznetsova et al., 2007).

UsNeA SUbFLORIDANA Stirt. - 2, 8, 25: Be, Pic.

VERRUCARIA DOLOSA Hepp - 10, 13.2: calst, det. JP, 2011 (H; Pykälä et al., 2012).

VERRUCARIA EPILITHEA Vain. - 13.2: calst, det. JP, 2011 (H; Pykälä et al., 2012).

VERRUCARIA ILLINOISENSIS Servit - 10: calst, det. JP, 2011 (H; Pykälä et al., 2012).

VERRUCARIA INASPECTA Servít - 13.2: calst, det. JP, 2011 (H; Pykälä et al., 2012).

VERRUCARIA MURALIS Ach. - 14: calst, det. JP, 2011 (H; Pykälä et al., 2012).

VERRUCARIA PILOSOIDES Servit - 13.2: calst, det. JP, 2011 (H; Pykälä et al., 2012).

VERRUCARIA TRANSFUGIENS Zschacke - 13.2: calst, det. JP, 2011 (H; Pykälä et al., 2012).

Violella fucata (Stirt.) T. Sprib. - 32, 33, 35: Be, Pic, Pis, 1Pis. Specimens contain atranorin and fumarprotocetraric acid, det. MK \& IS, 10.2011 .

VulPiCIDA PINASTRI (Scop.) J.-E. Mattsson \& M. J. Lai - 5.1, 7, 9, 10, 14, 16, 19, 22, 24, 25, 32, 33, 35-37: bark of coniferous and deciduous trees, dws (Ledum palustre, Vaccinium uliginosum) (Kuznetsova et al., 2007).

Xanthoria Parietina (L.) Th. Fr. - 1, 5.1, 9, 14, 15, 22, 31, 32, 39: Ali, Pot, lig (Kuznetsova et al., 2007).

XYLOGRAPHA VITILIGO (Ach.) J. R. Laundon - 13.1: lig.

XYLOPSORA FRIESII (Ach.) Bendiksby \& Timdal 2: Pic.

\section{DISCUSSION}

Despite being a rather small area, signs of former forest management and current recreational activity, the lichen biota of the Ragusha River valley is quite rich. A total of 211 lichenized, five lichenicolous and five lichen-related saprobic fungi were recorded from the studied area. Of them 207 species were found within the protected area. This species richness constitutes about $20 \%$ of the lichen biota of the whole Leningrad
Region. One species (Lecanora perpruinosa) is new for North-Western Russia, and six species (Lathargium fuscovirens, Pronectria erythrinella, Protoblastenia rupestris, Thelidium minutulum, T. zwackhii and Tremella hypogymniae) are published here for the first time for the LR. Also, new localities for seven Red List species were found.

Several taxa, viz. Acrocordia cavata, Collema subnigrescens, Cliostomum leprosum, Gyalecta truncigena, Hypogymnia vittata, Lobaria pulmonaria, Nephroma parile and Sclerophora pallida are considered to be habitat specialists, while Alectoria sarmentosa subsp. sarmentosa, Arthonia helvola, Bacidia rubella, Chaenotheca brachypoda, Cladonia norvegica, Leptogium saturninum, Scytinium lichenoides and S. teretiusculum are indicator species of old-growth forests in biologically valuable forests in NorthWestern European Russia (Andersson et al., 2009). Six of them inhabit riparian alder-elm forests with sporadic old elms. Other species are scattered throughout the protected area and are not concentrated enough to indicate biologically valuable forests.

Though the majority of recorded lichen species are common in the LR, a part of them - calcicolous lichens - is noteworthy for the region, where calcicolous lichens are sparse due to rarity of carbonate rocks. In total 117 species were recorded on natural and artificial (mainly concrete) carbonate substrates in St. Petersburg and the LR (e.g. Pykälä et al., 2012; Stepanchikova et al., 2016), of which only 45 are strictly calcicolous. In total 21 species were found only on limestones in the Ragusha River valley, even though walls of the canyon and large blocks of limestone at the bottom of the canyon were not inhabited by lichens. Most calcicolous species were found on small stones at the edge of water or subjected to periodical submersion. Four species found on carbonate rocks (Lecanora crenulata, $L$. dispersa, Verrucaria dolosa, $V$. muralis) are widespread in St. Petersburg and the LR, while eleven taxa are known only from the valley of the Ragusha River, and six further taxa - from a limited number of localities (2-8) in the LR.

\section{ACKNOWLEDGMENTS}

The authors are sincerely grateful to I. M. Brodo, M. Kukwa, J. Pykälä, I. S. Stepanchikova, M. Westberg and L. Yakovchenko for identification 
and revision of some specimens. The first author would like to thank Mikhail Bass as well as study group of the Laboratory of Ecology and Biomonitoring (St. Petersburg City Palace of Youth Creativities) for great help during the field trips. We are grateful to anonymous reviewer for valuable comments on the manuscript. O. W. Purvis is cordially thanked for linguistic corrections. The present study was carried out within the framework of the research project (no. 01201255601) of the Komarov Botanical Institute of the Russian Academy of Sciences. Financial support to the Russian authors was given by the Russian Foundation for Basic Research (14-04-01411). The research of the third author was supported by grant 1.37.151.2014 of Saint-Petersburg State University.

\section{REFERENCES}

Ābolina, A., Piterāns, A. \& Bambe, B. 2015. Latvias kुērpji un sūnas. Taksonu saraksts. (In Latvian). Salaspils. 213 pp.

Andersson, L., Alexeeva, N. \& Kuznetsova, E. (eds). 2009. Survey of biologically valuable forests in North-Western European Russia. Vol. 2. Identification manual of species to be used during survey at stand level. (In Russian). St. Petersburg. 258 pp.

Fadeeva, M. A., Golubkova, N. S., Vitikainen, O. \& Ahti, T. 2007. Conspectus of lichens and lichenicolous fungi of the Republic of Karelia (In Russian, English summary). Petrozavodsk. 194 pp.

Hermansson, J., Pystina, T. N., Owe-Larsson, B. \& Zhurbenko, M. 2006. Lichens and lichenicolous fungi of Pechero-Ilychsky Reserve. (In Russian). Flora and fauna of Reserves 109: 1-79.

Kataeva, O. A. 2009. Lichens and lichenicolous fungi (In Russian). In: Cadaster of flora of Novgorod Region. Veliky Novgorod. Pp. 247-254.

Kotkova, V. M. 2003. Aphyllophoraceous fungi of the "Ragusha River" Reserve and its Vicinity (Leningrad Region). (In Russian). Mycology and Phytopathology 37(4): 48-56.

Kukwa, M. 2009. The lichen genus Ochrolechia in the Baltic countries. Folia Cryptogamica Estonica 46: 67-74.

Kuznetsova, E., Ahti, T. \& Himelbrant, D. 2007. Lichens and allied fungi of the Eastern Leningrad Region. Norrlinia 16: 1-62.

Kuznetsova, E. S., Motiejūnaitè, J., Stepanchikova, I. S., Himelbrant, D. E. \& Czarnota, P. 2012. New records of lichens and allied fungi from the Leningrad Region, Russia. III. Folia Cryptogamica Estonica 49: 31-38.

Motiejūnaitè, J. 1999. Checkist of lichens and allied fungi of Lithuania. Botanica Lithuanica 5(3): 251-269.
Motiejūnaite, J. 2002. Diversity of lichens and lichenicolous fungi in the transboundary region of Marijampole district (southern Lithuania). Botanica Lithuanica 8(3): 277-294.

Motiejūnaitė, J. \& Andersson, L. 2003. Contribution to the Lithuanian flora of lichens and allied fungi. Botanica Lithuanica 9(1): 71-88.

Motiejūnaite, J. \& Grochowski, P. 2014. Miscellaneous new records of lichens and lichenicolous fungi. Herzogia 27(1): 193-198. http://dx.doi. org/10.13158/heia.27.1.2014.193

Muchnik, E. \& Śliwa, L. 2011. New records of Lecanora percrenata, with notes on other members of $L$. dispersa group in Ryazan Region (Russia). Polish Botanical Journal 56(1): 89-93.

Nordin, A., Moberg, R., Tonsberg, T., Vitikainen, O., Dalsätt, A., Myrdal, M., Snitting, D. \& Ekman, S. 2011. Santesson's Checklist of Fennoscandian Lichen-forming and Lichenicolous Fungi. Ver. April 29, 2011. http:130.238.83.220/santesson/home. php (20 March 2016).

Noskov, G. A. \& Botch, M. S. (eds). 1999. Red Data Book of Nature of the Leningad Region. Vol. 1. Protected areas. St. Petersburg. 352 pp.

Notov, A. A., Himelbrant, D. E. \& Urbanavichus, G. P. 2011. The list of lichens and allied fungi of Tver Region. (In Russian). Tver. 124 pp.

Orange, A., James, P. W. \& White, F. J. 2001. Microchemical methods for the identification of lichens. London. $101 \mathrm{pp}$.

Pykälä, J., Stepanchikova, I. S., Himelbrant D. E., Kuznetsova, E. S. \& Alexeeva, N. M. 2012. The lichen genera Thelidium and Verrucaria in the Leningrad Region (Russia). Folia Cryptogamica Estonica 49: 45-57.

Randlane, T., Saag, A. \& Suija, A. 2015. Lichenized, lichenicolous and allied fungi of Estonia. Ver. December 31, 2015. http://esamba.bo.bg.ut.ee/ checklist/est/home.php (20 March 2016).

Red Data Book of Russian Federation (Plants and Fungi). 2008. (In Russian). Moscow. 855 pp.

Stepanchikova, I. S., Kuznetsova, E. S. \& Himelbrant, D. E. 2009. New records of lichens and allied fungi from the Eastern Leningrad Region. Folia Cryptogamica Estonica 46: 75-78.

Stepanchikova, I. S., Kukwa, M., Kuznetsova, E. S., Motiejūnaitè, J. \& Himelbrant, D. E. 2010. New records of lichens and allied fungi from the Leningrad Region, Russia. Folia Cryptogamica Estonica 47: 77-84.

Stepanchikova, I. S., Tagirdzhanova, G. M. \& Himelbrant, D. E. 2013. The lichens and allied fungi of the Smorodinka River valley (Leningrad Region). (In Russian, English summary). Novitates Systematicae Plantarum Non Vascularum 47: 262-278.

Stepanchikova, I. S., Kuznetsova, O. A., Himelbrant, D. E. \& Kuznetsova, E. S. 2016. Lichen diversity on carbonate stone substrates in St. Petersburg, Russia: a review. Biogenic-Abiogenic Interactions in Natural and Anthropogenic Systems. Heidelberg. 
Pp. 403-413. http://dx.doi.org/10.1007/978-3319-24987-2_31

Tagirdzhanova, G. M., Kataeva, O. A. \& Stepanchikova, I. S. 2014. New lichen records from the Novgorod Region, Russia. Folia Cryptogamica Estonica 51: 103-107. http:/ /dx.doi.org/10.12697/ fce.2014.51.11

Tzvelev, N. N. (ed.). 2000. Red Data Book of nature of the Leningrad Region. Vol. 2. Plants and fungi. St. Petersburg. 672 pp.
Wainio, E. 1878. Lichenes in viciniis Viburgi observati. Meddelanden Societatis pro Fauna et Flora Fennica 2: 35-72.

Zavarzin, A. A., Katenina, O. A., Kotlov, Yu. V. \& Sokolova, S. V. 1999. Lichens of St. Petersburg and Leningrad Region. In: Biodiversity of the Leningrad Region (Algae. Fungi. Lichens. Bryophytes. Invertebrates. Fishes and pisciformes). (In Russian, English summary). Transactions of St. Petersburg Naturalists Society. Series 6. 2: 205-260. 